Debatte

2020, Band 8, Heft 3

Seiten 129-136

zeitschrift-suburban.de

10.36900/suburban.v8i3.629

\section{Rückschritte in längst überwunden geglaubte Ausbeutungs- und Unsicherheitsmuster}

Kommentar zu Matthias Bernt und Andrej Holm „Die Ostdeutschlandforschung muss das Wohnen in den Blick nehmen“
Matthias Bernt, Andrej Holm: ,Die Ostdeutschlandforschung muss das Wohnen in den Blick nehmen'

Kommentare von: Barbara Schönig, Carsten Keller, Carmen Leidereiter, Dieter Rink, Felix Ringel

Replik von:

Matthias Bernt, Andrej Holm

Der große Beitrag des Aufschlags von Bernt und Holm (2020) liegt darin, dem viel beschriebenen Abwertungsgefühl ostdeutscher Menschen eine materielle Basis real existierender Ungleichheiten nachzuweisen. Dadurch werden Ungleichheitsempfindungen von einem Status puren Affekts zu statistisch belegbaren Lebensrealitäten erhoben. Ich lese in diesem Aufschlag auch einen Impuls zur historisch-realistischen Analyse (Smith 2011) von Besitzund Machtverhältnissen, sowie deren Konsequenzen für die Bedingungen des täglichen (Über-)Lebens. Die Frage, der ich mich in diesem Kommentar widmen möchte, betrifft die konzeptuelle Anwendbarkeit und empirische Relevanz dieses Impulses für die ländlichen Räume Ostdeutschlands.

Meine Forschung im Kleinseengebiet Nordostdeutschlands lässt vermuten, dass sich die von den Autoren verfolgten Prozesse der Privatisierung, Spekulation und Enteignung im ruralen und urbanen Raum durchaus ähnlich artikulieren, da sie in vergleichbaren Strukturen materieller Schlechterstellung resultieren. Dem dieser Struktur folgenden Abwertungsgefühl kann man empirisch und analytisch nur gerecht werden, indem ein vollständiges Verständnis der Konsequenzen der seit der Wiedervereinigung entstandenen Besitz- und Eigentumsverhältnisse geschaffen wird, welches ländliche Räume einbeziehen muss. Im nächsten Schritt muss diese Analyse jedoch flankiert werden von einer Betrachtung der Akkumulationsprozesse von Grund und Boden in der longue durée sowie von einer Anerkennung der affektiven Spuren, die die rapiden Änderungen der Produktions- und Eigentumsstrukturen bei den Betroffenen hinterlassen.

Beginnend mit der Beobachtung, dass die der Privatisierung von Staatseigentum folgenden Marktprozesse nicht nur, wie von den Autoren erörtert, auf die Stadt- und Wohnungsentwicklung, sondern auch auf die Landentwicklung durchschlagen, nehme ich meine Fallstudie einer ländlichen Region circa 100 Kilometer nördlich von Berlin zum Anlass, drei der aufgezeigten Prozesse empirisch nachzuverfolgen: (1) Wert- und Preissteigerungen von Wohnbestand und Bauland; (2) Akkumulation landwirtschaftlicher Nutzflächen, besonderes der ehemaligen Subsistenzwirtschaft; (3) Kapitalbeteiligung nicht landwirtschaftlicher Investoren an landwirtschaftlichen Unternehmen durch renteerism und Spekulation. 


\section{Bauland und -substanz}

Wie auch im urbanen Raum lässt sich in der Seenplatte seit 2010 eine akute Steigerung der Kaufpreise von Bauland und Wohneigentum sowie der Quadratmeterpreise für Mietraum feststellen. Bedingt wird die Entwicklung einerseits durch den Zuzug von finanziell meist besser situierten Berlinern, Hamburgern und Westdeutschen allgemein, die die Gegend als Wochenendund Naherholungsgebiet nutzen, was Druck auf die Grundstücks-, Bau- und Mietpreise ausübt. Die auch in Städten beobachtete Zweckentfremdung von Wohnraum zur saisonalen touristischen Vermietung oder als Wochenenddomizil spielt ebenfalls eine Rolle (Glock et al. 2001), da sie sich verschärfend auf eine bereits angespannte Wohnsituation auswirkt.

Hierbei muss auch die regional geförderte touristische Entwicklung von ländlichen Regionen als Fortschrittsoption für den Servicesektor erwähnt werden (Harvey 2003). Regional artikuliert sich dieser Entwicklungsimpuls am häufigsten über die Nichtdurchsetzung beziehungsweise Nichtkontrolle existierender baulicher Genehmigungs- und Lizensierungsverfahren, durch die bauliches Vordringen selbst in Naturschutzgebiete indirekt ermöglicht wird. Ähnliches gilt für Verstöße gegen Bauentwicklungs- und Bebauungspläne, die über Jahrzehnte hinweg weder kontrolliert noch geahndet wurden, und daher eine touristische Entwicklung in einem „de facto regulationsfreiem Raum“ (Bundesministerium für Wirtschaft und Energie 2017: 33) ermöglichten.

Auf der anderen Seite wird hier, wie im urbanen Raum auch, Bauland und Baubestand als sogenannte wertbeständige Anlageoption zum Objekt von Kapital und Spekulation - eine Entwicklung, die sich insbesondere seit der letzten Finanzkrise beobachten lässt. Als beispielhaft kann der Aufkauf ganzer ehemaliger Bungalowsiedlungen und Jugendlandwohnheime zwecks (Luxus-)Sanierung und Neuverkauf angeführt werden.

\section{Subsistenz, Enteignung und die Bodenverwertungs- und Verwaltungsgesellschaft}

Weiterführend gilt es zu bemerken, dass die von den Autoren beschriebene staatlich subventionierte Spekulation und der ihr folgende Mehrwertabfluss nicht auf Bausubstanz und Bauland beschränkt ist. Vielmehr sind eben jene Prozesse auch in Bezug auf Acker, Grün- und Waldland zu beobachten. Institutionell muss hierbei die Rolle der Privatisierungspolitik der Bodenverwertungs und -verwaltungs GmbH (BVVG) für Ostdeutschland hervorgehoben werden. Was das Amt zur Regelung offener Vermögensfragen für den Immobilienbereich und die Treuhandanstalt für Betriebe waren, das war die BVVG für die Bodenprivatisierung: eine staatliche Institution, die ein Vergleichssystem zur Ermittlung von Kaufpreisforderungen für ehemalig volkseigenen Grund und Boden erstellte und anschließend Verkäufe abwickelte.

Ein kurzer historischer Abriss ist hier nötig. Die Kleinseeregion liegt im Gebiet des historischen Pommerns[1]. Bereits im geeinten Kaiserreich hatte Pommern eine Sonderstellung als erzkonservative Bastion sogenannter traditioneller Werte inne, in dem unfreie Bauern, Leibeigenschaft und 
feudale Großgrundbesitzverhältnisse bis weit in die 1930er Jahre Bestand hatten (Matthiesen 2000). Die Bodenreformen nach 1945 orientierten sich vor allem daran, möglichst vielen Bürger_innen Zugang zu landwirtschaftlicher Nutzfläche (LF) zu ermöglichen, und damit einen gewissen Grad der Selbstversorgung abzusichern (Mooser 1992). Der Zusammenschluss umverteilter Flächen in Landwirtschaftlichen Produktionsgenossenschaften(LPGs) erfolgte ab 1953 und wurde als Grundpfeiler der Planwirtschaft verstanden. In einem weiteren Schritt wurden ab 1952 Staatsforste, Seengebiete und Grünland verschiedenen Gemeinden zugeteilt. Zusätzlich zu den LPGs wurden einige dieser Grün- und Ackerflächen in Kleinststücke von oft nicht mehr als 1000 qm aufgeteilt und Familien beziehungsweise Haushalten zur Nutzung zugewiesen (Nölting 2009). Allerdings war dieser Zugang auf ein reines Nutzungsrecht beschränkt, während die Flächen formal Staatseigentum blieben und somit von der Privatisierungspolitik der BVVG ebenso betroffen waren wie Grün-, Acker- und Waldland.

Seit 1990 lässt sich vor allem ein Wandel der Eigentumsstruktur von Landflächen vermerken, indem Eigentum in immer höherem Maße auf GmbHs konzentriert ist, während Einzelpersonen- oder Familiengesellschaften abnehmen[2]. Die absoluten Kaufwerte je Hektar LF liegen in Ostdeutschland bei durchschnittlich 8.838 Euro, und damit circa 6o Prozent unter den in Westdeutschland geforderten Verkaufspreisen (Forster/Tietz 2013). Dieses Preisniveau macht LF in ähnlichem Maße sowohl zum Anlageobjekt als auch zum Objekt von Kapitalspekulation im Sinne des land[3] und green grabbing [4]. Als Resultat ist besonders in Mecklenburg-Vorpommern in den zehn Jahren seit der letzten Finanzkrise ein absoluter Kauf- und Wertanstieg von LF um 2.602 Euro pro Hektar zu verzeichnen - ein Trend, der Rückschlüsse auf die steigende Beteiligung nicht-landwirtschaftlicher Investoren an Landeigentum und Spekulation zulässt (ebd.: 45).

Dies hat unmittelbare Konsequenzen sowohl für die Menschen, die dieses Land gewohnheitsmäßig nutzen, als auch für die Rahmenbedingungen, unter denen sich die ländlichen Gebiete Ostdeutschlands entwickeln.

Einerseits wird durch Privatisierung die Überführung ehemals wertfreier öffentlicher Räume und Aspekte gesellschaftlichen Lebens in die marktwirtschaftliche Verwertungskette gesichert (enclosure of the commons). Ein hervorstechendes Beispiel aus meiner Feldforschung betrifft die Illegalisierung des Zugangs zu Wäldern, Gewässern und öffentlichem Boden für traditionelle Subsistenzaktivitäten (wie z. B. Holzsammeln, Angeln, Früchte- und Pilzsuche) sowie die Einführung empfindlicher Strafen bei Zuwiderhandlung.

Die Vehemenz, mit der eigentumsrechtliche Ansprüche durchgesetzt werden, steht in drastischem Kontrast zu den oft nachlässigen baurechtlichen Genehmigungsverfahren, wie sie oben Erwähnung finden. Sie widerspricht außerdem dem emischen Gerechtigkeitsempfinden derer, die auf Subsistenz als Überlebensstrategie angewiesen sind. Daran, dass also bei potenziell lukrativen Bauprojekten ein Auge zugedrückt wird, andere Verstöße gegen das Privat- oder Zivilrecht aber akribisch verfolgt werden, zeigt sich eine starke Parteinahme, die Unverständnis gegenüber der Durchsetzung geltender Regularien erzeugt. Verstärkt wird diese Gefühlsstruktur, weil hier ehemals staatlich ausdrücklich erwünschte (weil als die Gemeinschaft zementierend 
angesehene) Überlebenspraktiken faktisch illegalisiert werden. So wird die enclosure of the commons zum auch zeitgenössisch relevanten Muster der für den späten Neoliberalismus typischen Akkumulation durch Enteignung (Harvey 2003).

Andererseits werden durch massenhafte Landkäufe die Landbesitzverhältnisse Ostdeutschlands entlokalisiert und der Zugang zu Nutzfläche für Durchschnittsbürger_innen drastisch reduziert. Obwohl die BVVG den Kleinflächenbewirtschafter_innen ein Vorkaufsrecht eingeräumt hatte, konnten die wenigsten dieses in dem ihnen zugestandenen Zeitraum wahrnehmen. So fielen also auch Kleinststücke der von der BVVG vermittelten Privatisierung zu und die Möglichkeit zur Subsistenzlandwirtschaft verringerte sich drastisch.

Es wird klar, dass diese Entwicklung Subsistenzpraktiken hemmt und in Verbindung mit dem erschwerten Zugang zu Landflächen die Lebensverhältnisse verschlechtert: Mehr des sowieso schon knappen Einkommens muss zur Ernährung aufgewendet werden, während vorheriges Zusatzeinkommen aus dem Verkauf der Überschussproduktion wegfällt (Streitz 1995). Sofern diese Konsequenzen regionalpolitisch überhaupt zur Sprache kommen, werden sie als unintendierter Kollateralschaden grundsätzlich notweniger Reformen abgetan; sie erfahren also keine politische Kreditierung.

\section{Landwirtschaft, Kapitalbeteiligung und renteerism}

Durch die staatlich subventionierte Übernahme (Forster/Tietz 2013) gesamter ehemaliger LPGs sowie die steigende Kapitalbeteiligung nicht-landwirtschaftlicher Investoren an Agrarunternehmen wird nicht nur die Landeigentums-, und Zugangsstruktur, sondern auch deren Bewirtschaftung grundlegend verändert.

Obwohl für eine umfassende Genealogie dieser Entwicklung hier kein Platz ist, lässt sich zusammenfassend bemerken, dass die Kapitalbeteiligung nicht-landwirtschaftlicher und überregional agierender Investoren an landwirtschaftlichen Unternehmen in Ostdeutschland prozentual doppelt so hoch ist wie in den westlichen Bundesländern. In den drei Landkreisen der Seenplatte ist er so hoch wie sonst nur in Sachsen (Forster/Tietz 2013). Da die Besitzer dieses Landes in der Regel internationale Agrarunternehmen und Finanzspekulant_innen sind, zeichnet sich deren Bewirtschaftungsprämisse durch Gewinnmaximierung aus. Zwei generelle Trends sind zu observieren: Sofern die Eigentümer_innen hauptsächlich Agrarunternehmen sind, führt die Beteiligung nicht-landwirtschaftlicher Investoren zu monokulturalen und klimaschädlichen, aber gewinnsteigernden Bewirtschaftungssystemen. Die langfristigen ,kollateralen Konsequenzen“ solcher Systeme sind in der Fachliteratur minutiös erfasst.[5]

Im Falle von spekulativem Landbesitz und nicht selbst bewirtschaftenden Landbesitzer_innen sind die Konsequenzen komplexer. Beispielhalft lässt sich hier der Fall eines Investors mit Sitz in den Niederlanden anführen, dessen Hauptgesellschafter ausschließlich in Russland ansässig sind (IHKMV 2018: MV/72737HD/ccvks). 2005 erwarb diese Gesellschaft eine ehemalige LPG mit 100 Hektar. Aggressives Wachstum und das 
konsequente Überbieten aller konkurrierenden Landkäufer_innen sicherten der Gesellschaft eine Expansion auf 9000 Hektar im Jahr 2019, verteilt über ein Gebiet von circa 50 qkm. Nach eigenen Angaben ist das Hauptziel der Investitionsgesellschaft Kapitalsicherheit, wobei die Gewinnmaximierung von Investitionen durch wertsteigernde regionale Intervention (z. B. Infrastrukturprojekte oder Umwandlung in Bau- oder Gewerbeland) ausdrücklich als erwünscht beschrieben wird.

Sofern massive Wertsteigerungen nicht abzusehen sind, werden LF zur Bewirtschaftung verpachtet. In der Regel erfolgt dies an selbstgegründete, als Subunternehmen agierende Agrargenossenschaften. Im Falle der beschriebenen Investitionsgesellschaft war es mir nicht möglich, Einsicht in oder Aufschluss über die Eigentums-, Beteiligungs- oder Anteilshalter_innen der offiziell als Subunternehmen der Gesellschaft beschriebenen Agrargenossenschaft zu erhalten. Sicher ist jedoch, dass die Genossenschaft selbst nur einen Bruchteil (ca. zehn Prozent) der gepachteten LF bewirtschaftet, während der Rest in einem undurchsichtigen Netz an Sub- und Pachtverträgen unterverpachtet wird.

Diesen Pachtbauern zufolge haben sich die Pachtpreise über zehn Jahren vervierfacht, während die Marktpreise für landwirtschaftliche Erzeugnisse kontinuierlich sanken. Weiterhin ist festzustellen, dass sich die verbliebenen Bauern in Miet- und Pachtverhältnissen an ebenjenen Flächen bedienen müssen, die sie vormals zu verkaufen gezwungen waren. Die Landwirte werden somit nicht nur finanziell zahlungspflichtig: Sie betätigen sie sich nicht länger als autonom agierende, auf Subsistenz oder Planwirtschaft erpichte Unternehmer, sondern werden entweder zu contract farmern für international agierende Großunternehmen oder zu Herstellern von regionalen cash crops wie Spargel, Erdbeeren oder den Ausgangstoffen für Biodiesel. In beiden Fällen greifen sie für Ernte und Verpackung auf saisonale Arbeitskräfte und Tagelöhner zurück.

In diesem Beispiel, wie in vielen anderen Fällen spekulativen Landbesitzes durch nicht-landwirtschaftliche Investoren, wird Wertschöpfung überregional betrieben und werden Zins- und Kapitalgewinne aus Spekulation dem internationalen Finanzmarkt beziehungsweise der weiteren Privatisierung zugeführt, sodass kaum regionale Investitionen erfolgen. In der Folge entstehen, keine vier Generationen nach Vergesellschaftung des Großgrundbesitzes, neue quasi-feudale Eigentums- und Pachtverhältnisse von Grund und Boden, in denen international agierende Finanzgesellschaften fast landlosen ehemaligen Bauern und Arbeitern gegenüberstehen.

Aus Sicht der sie konfrontierenden Menschen ist der einzige substanzielle Unterschied dieser neuen Besitzer zu den ehemaligen Großgrundherren jener, dass sie gesichtslose Entitäten sind, die nicht einmal um persönliche Gefallen oder Patronagen gebeten werden können. Stattdessen argumentieren und agieren Investor_innen und die sie vertretenden Agrarunternehmen, wie Ihre historischen Vorbilder auch, mit dem ewigen Zwang der alternativlosen Wirtschaftlichkeit. Dabei werden mit ehemaligem Subsistenzland international erhebliche Gewinne einfahren. Konfrontiert werden diese neuen Großgrundbesitzer, wenn überhaupt, von ehemals autonomen Landwirten, die zu contract farmern, saisonalen Arbeitskräften und Tagelöhnern degradiert oder zum Abwandern ,ermuntert' wurden. 


\section{Resümee und Offene Fragen}

Zusammenfassend wird so eine Landbevölkerung geschaffen, deren einzige Überlebenschance erneut ausschließlich in der Vermarktung ihrer Arbeitskraft liegt, nachdem Subsistenzpraktiken und Zugang zu Land vereitelt wurden. In der Summe werden diese Entwicklungen zu Recht als genau der Rückschritt empfunden, der sie auch sind: Eine Wiederkehr längst überwunden geglaubter Ausbeutungs-, Ungerechtigkeits- und Unsicherheitsmuster, die strukturell eine dauerhafte Schlechterstellung darstellen. Die grundgesetzlich verankerte Gleichwertigkeit der Lebensverhältnisse im Bundesgebiet bleibt somit nicht nur aus, sondern wird verstärkt strukturell unterminiert.

Nach dieser knappen Erörterung der Artikulation neoliberaler Prozesse im ländlichen Raum sowie der lebenswidrigen Konsequenzen dieser Prozesse möchte ich noch einige der unerlässlichen Fragen aufzeigen, die sich aus diesen Einsichten ergeben:

Erstens wurden durch die BVVG sowie die selektive Duldung beziehungsweise drastische Ahndung widerrechtlichen Verhaltens seit der Wiedervereinigung auch im ländlichen Raum Prozesse der Ungleichheiten und ein daraus resultierendes Machtgefälle staatlich institutionalisiert. Die von Bernt und Holm beschriebenen urbanen Prozesse finden also im ländlichen Raum ein strukturelles Pendant. Diese Einsicht ermöglicht es uns, zu verstehen, wie die agency ostdeutscher Menschen machtpolitisch prädeterminiert ist. Basierend auf Untersuchungen anderer postsozialistischer Regionen (Materka 2017; Shevchenko 2009) ist jedoch davon auszugehen, dass diese anscheinende Pfadabhängigkeit und die ihr folgende Ungerechtigkeit nicht still hingenommen werden: Was also sind die Möglichkeiten für subversives Handeln? Wie werden die institutionellen Rahmenbedingungen und Machtstrukturen unterminiert oder zumindest umgangen? Welche (informellen) Überlebensstrategien und Wohnlösungen stehen heute offen?

Zweitens hinterlassen die rapiden Änderungen der Produktions- und Eigentumsstrukturen innerhalb von nur drei Generationen (vom Großgrundbesitz zu Allgemeingut und zurück zu Großgrundbesitz, oder von der Wohnsicherheit in die Wohnunsicherheit) emotionale, affektive Spuren, deren Ausdruck nicht einfach mit Referenz zum Tellerrand der Subjektperspektive abgetan werden kann (Phillips 2005)[6]. Was es braucht, ist vielmehr eine institutionell-politische Perspektive, die flankiert wird von einer Affektanalyse der Subjektivitäten, die durch ein Existieren in ebenjener permanenten Unsicherheit produziert werden (Federici 2012; Fassin 2009; Arendt 1958). Was geschieht mit Identitäten, Subjektivitäten und Sozialität, wenn die teleologische Sicherheit, in der alles für immer war (Yurchak 2006) durch die hochvolatilen Mechanismen flexibler Akkumulation ersetzt wird?

Drittens muss die Tatsache, dass es Kohls viel beschriebene und bald karikierte blühende Landschaften - eine Metapher für das im Grundgesetz festgeschriebene Anrecht auf eine Gleichwertigkeit der gesamtdeutschen Lebensverhältnisse - auch 30 Jahre nach der Wiedervereinigung nicht gibt, anerkannt und kreditiert werden. Die Problematik der Wertung heterogener Verhältnisse und nicht-linearer Lebenserfahrungen kulminiert vielleicht in Madina Tlostanovas (2012, 2015) provozierender Frage, ob das Postsozialistische sprechen und denken kann. Welche Möglichkeiten 
bestehen also für eine gesamtgesellschaftliche Anerkennung teils traumatischer Transformationserfahrungen (Suchland 2001), real existierender Ungleichheit der Lebensverhältnisse und des Wiedererstarkens tiefer Ausbeutungsstrukturen?

\section{Endnoten}

[1] Sie ist heute jedoch zwischen drei Landkreisen aufgeteilt: Ostprignitz-Ruppin und Oberhavel in Brandenburg sowie Mecklenburg-Strelitz.

[2] Oftmals wurden LPGs zumindest mittelfristig von den sie bewirtschaftenden Bauern übernommen, gingen bankrott und wurden dann von der BVVG bis zum Verkauf verwaltend übernommen.

[3] Grob übersetzt bedeutet das ,Landraub', neue Landnahme (vgl. auch Dörre 2017). Eine präzise deutsche Übersetzung für land grabbing fehlt bisher. Der Weltagrarbericht definiert land grabbings als ,großflächige Käufe hauptsächlich von privaten, aber auch staatlichen Investoren und Agrarunternehmen, die Agrarflächen kaufen oder langfristig pachten, um sie in eigener Regie zur Herstellung von Agrarrohstoffen zu nutzen. Dabei bewegen [sie] sich oft in Grauzonen des Rechts [...] zwischen traditionellen Landrechten und modernen Eigentumsverhältnissen" (https://www.weltagrarbericht.de/themen-desweltagrarberichts/landgrabbing.html, letzter Zugriff am 8.10.2020).

[4] Wörtlich übersetzt, grüner Raub'. Eine sinngemäße Übersetzung wäre, grüne Landnahme', eine Mischform, grüner (Land-)Raub'. In Anlehnung an land grabbing definieren Farihead, Leach und Scone (2012) green grabbing als die großflächige Privatisierung von Land, Ressourcen und Wasser unter dem Vorwand des ökologischen Schutzes, z. B. der Artenvielwalt, Kohlenstoffbindung, Biodiversität oder Ökotourismus. Green grabs gefährden traditionelle Landrechte und Subsistenzpraktiken in ähnlichem Maße wie land grabbing.

[5] Gemeint sind Desertifikation, ökologische Armut, reduzierter Wildtierbestand bis hin zur Unbewohnbarkeit ganzer Landstriche, wobei diese sozialen und ökologischen Konsequenzen meistens an Privatpersonen und staatliche Institutionen externalisiert werden können.

[6] Ost-West-Unterschiede zeigen sich unter anderem auch bezüglich der (mentalen) Gesundheit: So ist zum Beispiel die Prävalenz von Angststörungen und Depression in Ostdeutschland um 6o Prozent höher als im Westen (Prütz et al. 2014).

\section{Autor_innen}

Carmen Leidereiter ist Soziologin und Sozialanthropologin und forscht zur Restrukturierung von Arbeit und Kapital in Krisen sowie zur Rekonfiguration alltäglicher ökonomischer Haushaltspraktiken und Moralvorstellungen.

c.leidereiter@gmail.com

\section{Literatur}

Arendt, Hannah (1958): The Human Condition. Chicago: University of Chicago Press.

Bundesministerium für Wirtschaft und Energie (BMWi) (2017): Tourismuspolitischer Bericht der Bundesregierung, 18. Legislaturperiode.

Bernt, Matthias / Holm, Andrej (2020): Die Ostdeutschlandforschung muss das Wohnen in den Blick nehmen. Plädoyer für eine neue politisch-institutionelle Perspektive auf ostdeutsche Städte. In: sub \urban. zeitschrift für kritische stadtforschung 8/3, 97-114.

Dörre, Klaus (2017): Die neue Landnahme. Dynamiken und Grenzen des Finanzmarktkapitalismus. In: Klaus Dörre / Stephan Lessenich / Hartmut Rosa (Hg.), Soziologie - Kapitalismus - Kritik. Eine Debatte. Frankfurt am Main: Suhrkamp. 
Fairhead, James / Leach, Michael / Scoones, Ian (2012): Green Grabbing: A new Appropriation of Nature? In: Journal of Peasant Studies 39/2, 237-261.

Fassin, Diddier (2009): The Empire of Trauma: An Inquiry into the Condition of Victimhood. Princeton: Princeton University Press.

Federici, Silvia (2012): Revolution at Point Zero: Housework, Reproduction, and Feminist Struggle. Oakland: PM Press.

Forster, Bernd / Tietz, André (2013): Kapitalbeteiligung nichtlandwirtschaftlicher und überregional ausgerichteter Investoren an landwirtschaftlichen Unternehmen in Deutschland. Braunschweig: Thünen-Institut.

Glock, Birgit / Häußermann, Harmut / Keller, Carsten (2001): Die sozialen Konsequenzen der Restitution von Grundeigentum in Deutschland und Polen. In: Berliner Journal für Soziologie 11, 533-550.

Harvey, David (2003): The New Imperialism. Oxford: Oxford University Press.

Materka, Edyta (2017): Dystopian Provocateurs: Peasants, State and Informality in the Polish-German Borderlands. Indianapolis: Indiana University Press.

Matthiesen, Helge (2000): Greifswald in Vorpommern: Konservatives Milieu im Kaiserreich, in Demokratie und Diktatur; 1900-1990. Beiträge zur Geschichte des Parlamentarismus und der politischen Parteien. Düsseldorf: Droste.

Mooser, Josef (1992): Preußische Agrarreformen, Bauern und Kapitalismus. Bemerkungen zu Hartmut Harnischs Buch „Kapitalistische Agrarreform und Industrielle Revolution“. In: Geschichte und Gesellschaft 18/4, 533-554.

Nölting, Bernward (2009): Regionaler Wohlstand als Ziel der ländlichen Entwicklung in Ostdeutschland. In: Rainer Friedel / Edmund A. Spindler (Hg.), Nachhaltige Entwicklung ländlicher Räume. Wiesbaden: VS.

Phillips, Sarah D. (2005): Postsocialism, Governmentality, and Subjectivity: An Introduction. In: Ethnos 70/4, 437-442.

Prütz, Franziska / Rommel, Alexander / Kroll, Lars Eric / Lampert, Thomas (2014): GBE Bericht: 25 Jahre nach Fall der Mauer: Regionale Unterschiede in der Gesundheit. Berlin: Robert Koch Institut.

Shevchenko, Olga (2009): Crisis and the Everyday in Postsocialist Moscow. Indianapolis: Indiana University Press.

Smith, Gavin (2011): Selective Hegemony and Beyond-Populations with „No Productive Function": A Framework for Enquiry. In: Identities: Global Studies in Culture and Power 18/1, 2-38.

Streitz, Michel (1995): Cultivateur du Mecklembourg: Survui d'un Savoir Faire. In: Études rurales 138/140, 185-194.

Suchland, Jennifer (2001): Is Postsocialism Transnational? In: Signs: Journal of Women in Culture and Society 36/4, 837-62.

Tlostanova, Madina V. (2012): Postsocialist $\neq$ Postcolonial? On Post-Soviet Imaginary and Global Coloniality. In: Journal of Postcolonial Writing 48/2, 130-142.

Tlostanova, Madina V. (2015): Can the Post-Soviet Think? On Coloniality of Knowledge, External Imperial and Double Colonial Difference. In: Intersections. East European Journal of Society and Politics 1/2, 38-58.

Yurchak, Alexej (2006): Everything Was Forever, Until It Was No more: The Last Soviet Generation. Princeton: Princeton University Press. 